\title{
Removal of Chromium (III) from Water by Using Modified and Nonmodified Carbon Nanotubes
}

\author{
Muataz Ali Atieh,, ${ }^{1,2}$ Omer Yahya Bakather, ${ }^{1}$ Bassam S. Tawabini, ${ }^{3}$ Alaadin A. Bukhari, ${ }^{4}$ \\ Mazen Khaled, ${ }^{5}$ Mamdouh Alharthi, ${ }^{1}$ Mohammed Fettouhi, ${ }^{5}$ and Faraj Ahmad Abuilaiwi ${ }^{2,6}$ \\ ${ }^{1}$ Chemical Engineering Department, King Fahd University of Petroleum \& Minerals, Dhahran 31261, Saudi Arabia \\ ${ }^{2}$ Center of Research Excellence in Nanotechnology (CENT), King Fahd University of Petroleum \& Minerals, \\ Dhahran 31261, Saudi Arabia \\ ${ }^{3}$ Earth Sciences Department, King Fahd University of Petroleum \& Minerals, KFUPM P.O. Box 952, Dhahran 31261, Saudi Arabia \\ ${ }^{4}$ Center for Environment \& Water, King Fahd University of Petroleum \& Minerals, Dhahran 31261, Saudi Arabia \\ ${ }^{5}$ Chemistry Department, King Fahd University of Petroleum \& Minerals, Dhahran 31261, Saudi Arabia \\ ${ }^{6} \mathrm{Hafr}$ Al-Batin Community College, King Fahd University of Petroleum \& Minerals, Hafr Al-Batin 31991, Saudi Arabia
}

Correspondence should be addressed to Bassam S. Tawabini, bassamst@kfupm.edu.sa

Received 16 October 2009; Revised 21 January 2010; Accepted 9 March 2010

Academic Editor: Hongchen Chen Gu

Copyright (C) 2010 Muataz Ali Atieh et al. This is an open access article distributed under the Creative Commons Attribution License, which permits unrestricted use, distribution, and reproduction in any medium, provided the original work is properly cited.

\begin{abstract}
This study was carried out to evaluate the environmental application of modified and nonmodified carbon nanotubes through the experiment removal of chromium trivalent (III) from water. The aim was to find the optimal condition of the chromium (III) removal from water under different treatment conditions of $\mathrm{pH}$, adsorbent dosage, contact time and agitation speed. Multi wall carbon nanotubes (MW-CNTs) were characterized by field emission scanning electron microscopy (FE-SEM) and transmission electron microscopy (TEM). The diameter of the carbon nanotubes produced varied from 20-40 nm with average diameter of $24 \mathrm{~nm}$ and 10 micrometer in length. Adsorption isotherms were used to model the adsorption behavior and to calculate the adsorption capacity of the absorbents. The results showed that, $18 \%$ of chromium (III) removal was achieved using modified carbon nanotubes (M-CNTs) at pH 7, $150 \mathrm{rpm}$, and 2 hours for a dosage of $150 \mathrm{mg}$ of CNTs. The removal of Cr (III) is mainly attributed to the affinity of chromium (III) to the physical and chemical properties of the CNTs. The adsorption isotherms plots were well fitted with experimental data.
\end{abstract}

\section{Introduction}

The pollution of water resources due to the disposal of heavy metals has been causing worldwide concern. The main sources of these metals are mining, metallurgical and chemical manufacturing, tannery, battery manufacturing industries, and fossil fuel; the modern chemical industry is based largely on catalysts, many of which are metals or metal compounds; production of plastics, such as polyvinyl chloride, involves the use of metal compounds, particularly as heat stabilizers and so forth. The effects of heavy metals such as copper, lead, zinc, mercury, chromium and cadmium on human health have been investigated extensively. Lead is ubiquitous in the environment and is hazardous at high levels. Long-term drinking water containing high level of lead will cause the nervous system damage, renal kidney disease, mental retardation, cancer and anaemia [1]. Lead is nonbiodegradable and, therefore, must be removed from water [2]. Many methods have been developed and used to remove metal ions from wastewater such as granulated activated carbon [3], fly ash [4], peat [5], recycled alum sludge [6], peanut hulls [7], resins [8], kaolinite [9], manganese oxides [10], zeolite [11], biomaterials [12, 13]. However, the removal efficiencies of metal ions of these adsorbents were low. Therefore, researchers carried out investigation for new promising adsorbents [14]. Carbon nanotubes (CNTs), a member in carbon family, have novel properties that make them potentially useful in many applications in 
nanotechnology, electronics, optics, water treatment, and other fields of materials science. Since their discovery in 1991 [15], carbon nanotubes (CNTs) have attracted considerable researchers' interest due their exceptional mechanical electrical properties, highly chemical stability, and large specific area [16]. Multiwalled carbon nanotubes (MWCNT) were previously used for removal of metal ions, such as lead, copper, cadmium, silver, and nickel [17]. Li et al. reported that carbon nanotubes have high adsorption efficiency for lead removal from water and the adsorption of lead is higher than copper and cadmium and significantly influenced by $\mathrm{pH}$ [18]. Hsieh and Horng reported that the adsorption capacity of CNTs grown on aluminum oxide, for $\mathrm{Pb}^{2+}$, $\mathrm{Cu}^{2+}$, and $\mathrm{Cd}^{2+}$ from solutions is superior to that of active carbon powders, commercial CNTs, and aluminum oxide [19]. Kandah and Meunier found that the adsorption of $\mathrm{Ni}^{2+}$ by oxidized CNTs is 1.24 times greater than commercial activated carbon called MINOTAUR [20]. Xu et al. reported that removal of $\mathrm{Pb}$ (II) is strongly dependent on $\mathrm{pH}$, ions strength and the type of foreign ions [21].

In this work the effect of modified and nonmodified carbon nanotubes were used to study the effect of these nanomaterilas on the removal the chromium (III) from water. The process parameters such as $\mathrm{pH}$, dosage of MWCNTs, agitation speed and contact time were investigated in order to maximize the removal of $\mathrm{Cr}$ (III).

\section{Experimental}

2.1. Production of Carbon Nanotubes. The experimental setup used to synthesise the Multi Wall Carbon Nanotubes is similar to that reported by Muataz et al. [22-27]. The Floating Catalyst Chemical Vapor deposition (FC-CVD) reactor has been used to produce CNTs. The production of CNTs in the present work has been conducted in a horizontal tubular reactor. The horizontal reactor is a quartz tube of $50 \mathrm{~mm}$ in diameter and $900 \mathrm{~mm}$ in length and heat by silicon carbide heating element. In this study benzene $\left(\mathrm{C}_{6} \mathrm{H}_{6} 99.5 \%\right.$ purity) was used as a hydrocarbon source, ferrocene (Fe $\mathrm{C}_{10} \mathrm{H}_{10} 98 \%$ purity), Hydrogen as carrier gas, and argon for flashing the air from the system. Several experiments were performed at the reaction temperature ranging from $500^{\circ} \mathrm{C}$ to $1200^{\circ} \mathrm{C}$. Other conditions like the reaction time (45 minute) and hydrogen flow rate $(300 \mathrm{ml} / \mathrm{min})$ were fixed.

The produced carbon nanotubes were characterized by using Field emission scanning electron Microscopy (FESEM) and Transmission Electron Microscopy (TEM) and the purity was measured by Thermogravimetric Analysis (TGA).

2.2. Oxidation of MWCNTs. Multiwall carbon nanotubes were purchased from Nanostructured \& Amorphous Materials, Inc. USA. The Purity of MWCNT is $>95 \%$, its outside and inside diameters are $10-20 \mathrm{~nm}$ and $5-10 \mathrm{~nm}$, respectively. The length of these MWCNTs is 10-30 um. Three hundred $\mathrm{ml}$ of a concentrated nitric acid of AnalaR (69\%) are added to $2 \mathrm{~g}$ of as-received MWCNT. The mixture is refluxed for 48 hours at $120^{\circ} \mathrm{C}$. After cooling to room temperature,

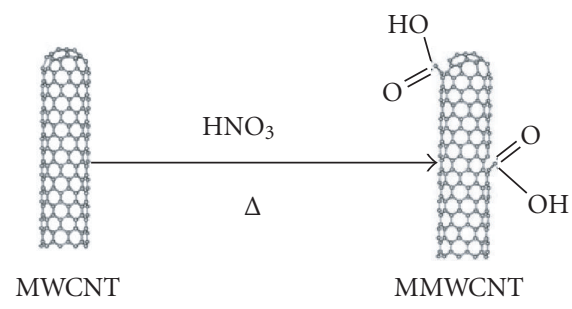

FIGURE 1: Chemical modification of carbon nanotubes (MWCNT) through thermal oxidation.

the reaction mixture is diluted with $500 \mathrm{ml}$ of deionised water and then vacuum-filtered through a filter paper $(3 \mu \mathrm{m}$ porosity). This washing operation is repeated until the $\mathrm{pH}$ becomes the same as deionsed water $\mathrm{pH}$ and is followed by drying in a vacuum oven at $100^{\circ} \mathrm{C}$. Such conditions lead to remove the catalysts from carbon nanotubes and opening the tube caps as well as the formation of holes in the sidewalls, followed by an oxidative etching along the walls with the concomitant release of carbon dioxide. This less vigorous conditions, minimized the shortening of the tubes and the chemical modification is then limited mostly to the opening of the tube caps and the formation of functional groups at defect sites along the sidewalls. The final products are nanotube fragments whose ends and sidewalls are decorated with various oxygen-containing groups (mainly carboxyl groups) (Figure 1). Moreover, the percentage of carboxylic functions on the oxidized MWCNT surface does not exceed $4 \%$ in the best cases, which corresponds to the percentage of MWCNT structural defects [28, 29].

2.3. Preparing the Stock Solution. The preparation of chromium stock solution is needed to produce stock solution with concentration, $1 \mathrm{mg} / \mathrm{L}$. Initially, the laboratory glass wares that were used in this project were rinsed with $2 \%$ of Nitric Acid. This step is very important to remove all the impurities on the glassware and to prevent further adsorption of chromium (III) on the wall of the glassware. The stock solution is prepared by adding $2 \mathrm{~mL}$ of chromium (III) from chromium (III) standard solution of concentration, $1000 \mathrm{mg} / \mathrm{L}$ into volumetric flask (2 L). Then, deionized water is added till the mark volumetric flask and mixed thoroughly using magnetic stirrer. The $\mathrm{pH}$ of The stock solution is adjusted by using 1.0 M Nitric Acid or $1.0 \mathrm{M} \mathrm{NaOH}$. Finally buffer solutions were added to maintain constant $\mathrm{pH}$ during the experimen. Multiwalled carbon nanotube (MWCNT) was $10-20 \mathrm{~nm}$ with main range of diameter $5-15 \mu \mathrm{m}, \geq 95 \%$ purity, 40-30 special area.

2.4. Batch Mode Adsorption Experiment. The experimen of the batch mode adsorption was conducted at room temperature to study the effect of initial solution $\mathrm{pH}$, CNTs dosage, contact time, and agitation speed on the adsorption of $\mathrm{Cr}$ (III) ions. Each experiment was conducted in volumetric flask and the initial and final concentrations of $\mathrm{Cr}$ (III) were analyzed by using Inductively Coupled Plasma (ICP). 
2.5. Adsorption Isotherms Models. Adsorption isotherms are mathematical models that describe the distribution of the adsorbate species between liquid and adsorbent, based on a set of assumptions that are mainly related to the heterogeneity/homogeneity of adsorbents, the type of coverage, and possibility of interaction between the adsorbate species. The Langmuir model assumes that there is no interaction between the adsorbate molecules and the adsorption is localized in a monolayer. The Freundlich isotherm model is an empirical relationship describing the adsorption of solutes from a liquid to a solid surface and assumes that different sites with several adsorption energies are involved. In order to model the adsorption behavior and calculate the adsorption capacity for the adsorbent, the adsorption isotherms will be studied. The Langmuir adsorption isotherm is perhaps the best known of all isotherms describing adsorption and it is often expressed as

$$
Q_{e}=\frac{X_{m} K C_{e}}{\left(1+K C_{e}\right)},
$$

where $Q_{e}$ : the adsorption density at the equilibrium solute concentration $C_{e}$ : (mg of adsorbate per g of adsorbent), $C_{e}$ : the equilibrium adsorbate concentration in solution $(\mathrm{mg} / \mathrm{l})$, $X_{m}$ : the maximum adsorption capacity corresponding to complete monolayer coverage ( $\mathrm{mg}$ of solute adsorbed per $\mathrm{g}$ of adsorbent), and $K$ : the Langmuir constant related to energy of adsorption ( $l$ of adsorbent per $\mathrm{mg}$ of adsorbate).

The above equation can be rearranged to the following linear form:

$$
\frac{C_{e}}{Q_{e}}=\frac{1}{X_{m} K}+\frac{C_{e}}{X_{m}} .
$$

The linear form can be used for linearization of experimental data by plotting $C_{e} / Q_{e}$ against $C_{e}$. The Langmuir constants $X_{m}$ and $K$ can be evaluated from the slope and intercept of linear equation.

In addition, we can describe adsorption with Langmuir if there is a good linear fit. If not then maybe some other model will work. Therefore, we can use Freundlich isotherm:

$$
Q_{e}=K_{F} C_{e}^{1 / n},
$$

where $Q_{e}$ is the adsorption density (mg of adsorbate per $\mathrm{g}$ of adsorbent), $C_{e}$ is the concentration of adsorbate in solution $(\mathrm{mg} / \mathrm{l}), K_{f}$ and $n$ are the empirical constants dependent on several environmental factors and $n$ is greater than one.

This equation is conveniently used in the linear form by taking the logarithm of both sides as

$$
\ln Q_{e}=\ln K_{f}+\frac{1}{n} \ln C_{e} .
$$

A plot of $\ln C_{e}$ against $\ln Q_{e}$ yielding a straight line indicates the confirmation of the Freundlich isotherm for adsorption. The constants can be determined from the slope and the intercept.

2.6. Kinetic Modeling. The study of sorption kinetics is applied to describe the adsorbate uptake rate and this rate evidently controls the residence time of adsorbate at solid liquid interface. In order to evaluate the mechanism of sorption of $\mathrm{Cr}$ (III) by the CNTs, the first-order equation, the pseudo-second-order rate equation and the second-order rate equation are calculated by the below shown equations, respectively:

$$
\begin{gathered}
\log \frac{q_{e}-q_{t}}{q_{e}}=-\frac{K_{L} t}{2.303}, \\
\frac{t}{q_{t}}=\frac{1}{2 K_{s} q_{e}^{2}}+\frac{t}{q_{e}}, \\
\frac{1}{q_{e}-q_{t}}=\frac{1}{q_{e}}+k t,
\end{gathered}
$$

where: $q_{e}$ : sorption capacity at equilibrium, $q_{t}$ : sorption capacity at time $(\mathrm{mg} / \mathrm{g}), K_{L}$ : the Lagergren rate constant of adsorption $(1 / \mathrm{min}), k$ : rate constant of the pseudo-secondorder sorption $\left(\mathrm{g} \cdot \mathrm{mg}^{-1} \cdot \mathrm{min}^{-1}\right), t$ : time $(\mathrm{min})$.

The linear plots of $\log \left(q_{e}-q_{t}\right)$ versus $t, t / q_{t}$ versus $t$ and $1 /\left(q_{e}-q_{t}\right)$ versus $t$ of the above equations, $q_{e}, K_{L}$, and $k$ can be determined from the slopes and intercepts.

\section{Results and Discussion}

3.1. Characterization of Carbon Nanotubes. High purity of multi wall carbon nanotubes were produced by chemical vapor deposition (CVD) technique. The produced carbon nanotubes were observed by suing field emission scanning electron microscopy (FE-SEM) and transmission electron microscopy (TEM). The diameter of the produced carbon nanotubes were varied from 20 to $40 \mathrm{~nm}$ with average diameter at $24 \mathrm{~nm}$ while the length of the CNTs was up to few microns. Figure 2(a) shows the SEM image of carbon nanotubes at low magnification. While Figure 3(b) shows the SEM image of carbon nanotubes at high magnification. From the SEM observation, the product is pure and only carbon nanotubes were observed.

TEM was carried out to characterize the structure of nanotubes (Figure 3). To prepare TEM samples, some alcohol was dropped on the nanotubes film, then, these films were transferred with a pair of tweezers to a carbon-coated copper grid. It is obvious from the images that all the nanotubes are hollow and tubular in shape. In some of the images, catalyst particles can be seen inside the nanotubes. TEM images indicate that the nanotubes are of high purity, with uniform diameter distribution and contain no deformity in the structure. While Figure 3(b) shows the High Resolution Transmission Electron Microscope (HRTEM) of the carbon nanotubes, it shows that a highly ordered crystalline structure of CNT is present.

3.2. Purity Measurement. The thermal analyses results of thermogravimetric (TG) and Derivative thermogravimetric (DTG) curves obtained for CNTs at heating rates $\left(10^{\circ} \mathrm{C}\right.$ /min) shown in Figure 4. The TG thermgrams was carried out in air and it was noted that there was some residual remains of the sample, when it was heated to about $900^{\circ} \mathrm{C}$. The residue appears reddish which shows that all the CNTs 


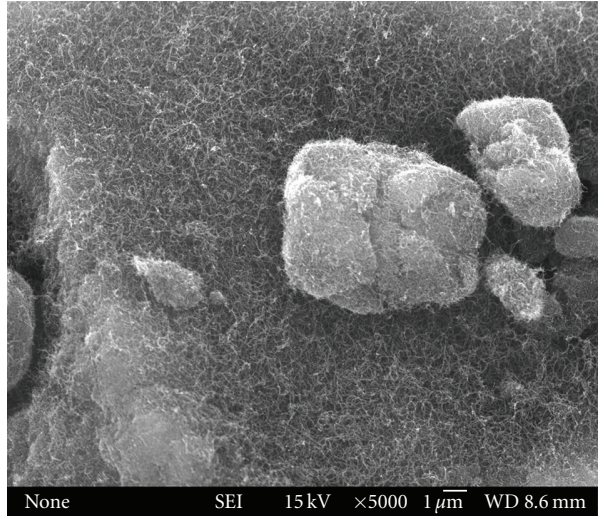

(a)

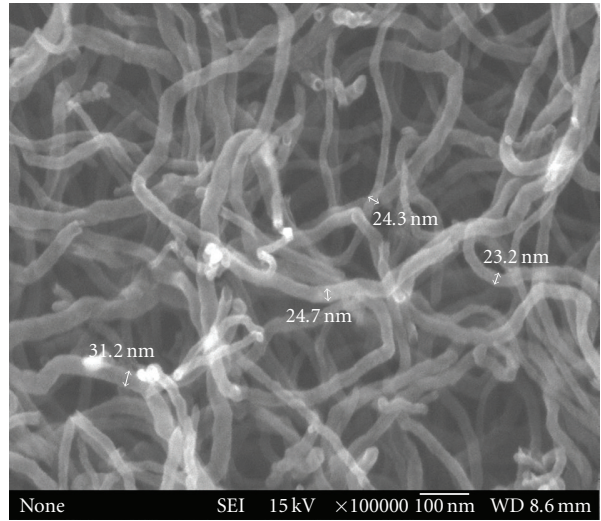

(b)

FIGURE 2: SEM Images of carbon nanotubes at (a) at low resolution and (b) at high resolution.

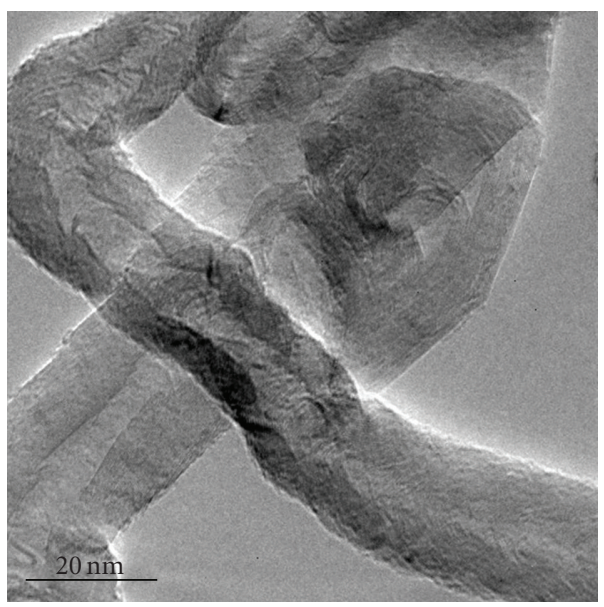

(a)

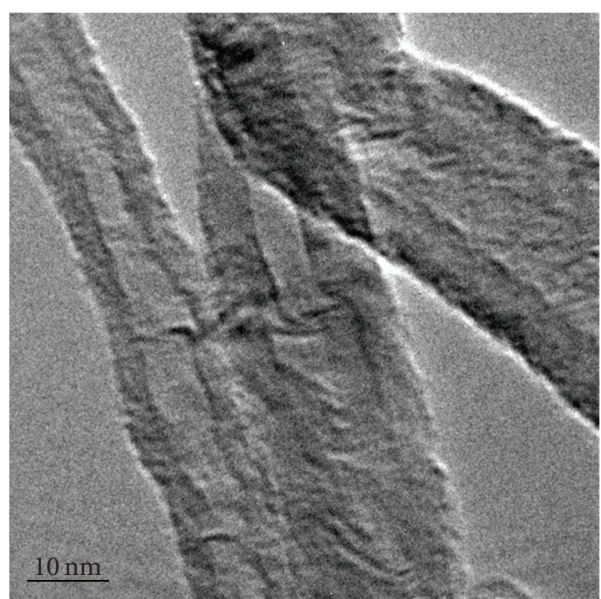

(b)

Figure 3: TEM Images of carbon nanotubes (a) at low resolution (b) at high resolution.

were oxidize leaving only the catalyst. It can be seen that this decomposition process is a single-stage decomposition reaction where the procedural decomposition temperatures are well defined. It shows that the initial degradation temperature $\left(T_{i}\right)$ was at $520^{\circ} \mathrm{C}$ while the maximum weight loss was at $\left(T_{m}\right) 650^{\circ} \mathrm{C}$ and the final degradation temperature was at $\left(T_{f}\right) 740^{\circ} \mathrm{C}$. The DTG is showing only one peak which in indicate that this material is pure.

3.3. Functionalization of CNTs with Carboxylic Functional Group (COOH). The as-received MWCNTs show one peak at around $1640 \mathrm{~cm}^{-1}$ (Figure 5) ascribed to the oscillation of carboxylic groups which moves to $1728 \mathrm{~cm}^{-1}$ associated with the stretch mode of carboxylic groups is observed in the IR spectrum of the acid-treated MMWNTs (Figure 5), indicating that carboxylic groups are formed and $\mathrm{C}=\mathrm{O}$ liaison of the carboxylic acid function due to the oxidation of some carbon atoms on the surface of the MWNTs by nitric acid. It should be noticed that the as-received MWCNTs were purified and a part of the catalytic metallic nanoparticles were possibly eliminated during the purification process cutting the nanotube cap. Thus, the presence of carboxylic groups in these commercial MWCNTs was expectable. The peak at $1554 \mathrm{~cm}^{-1}$ can be associated with the stretching of carbon nanotubes backbone. Increasing in the strength of the peak appearing at $1014 \mathrm{~cm}^{-1}$ is possibly associated with $\mathrm{O}-\mathrm{H}$ bending deformation in carboxylic acid groups whilst increased strength of the signal at $1168 \mathrm{~cm}^{-1}$ may be associated with $\mathrm{C}-\mathrm{O}$ stretching in the same functionalities. Moreover, it should be noted that there is no significant difference between the spectra of the samples before and after the $\mathrm{HNO}_{3}$ treatment. Two other peaks appearing between the region 1720.6 and $1630.2 \mathrm{~cm}^{-1}$, can be attributed to the carboxylic group incorporated onto the CNT as a result of the acid treatment with nitric acid. The peaks appearing at $2921.0 \mathrm{~cm}^{-1}$ and $3398.9 \mathrm{~cm}^{-1}$ are due to the carbonhydrogen bond stretch and hydroxyl group, respectively. The hydroxyl group can be attributed to the acid treatment of the CNT after production to remove the metal catalyst and purification purpose. 


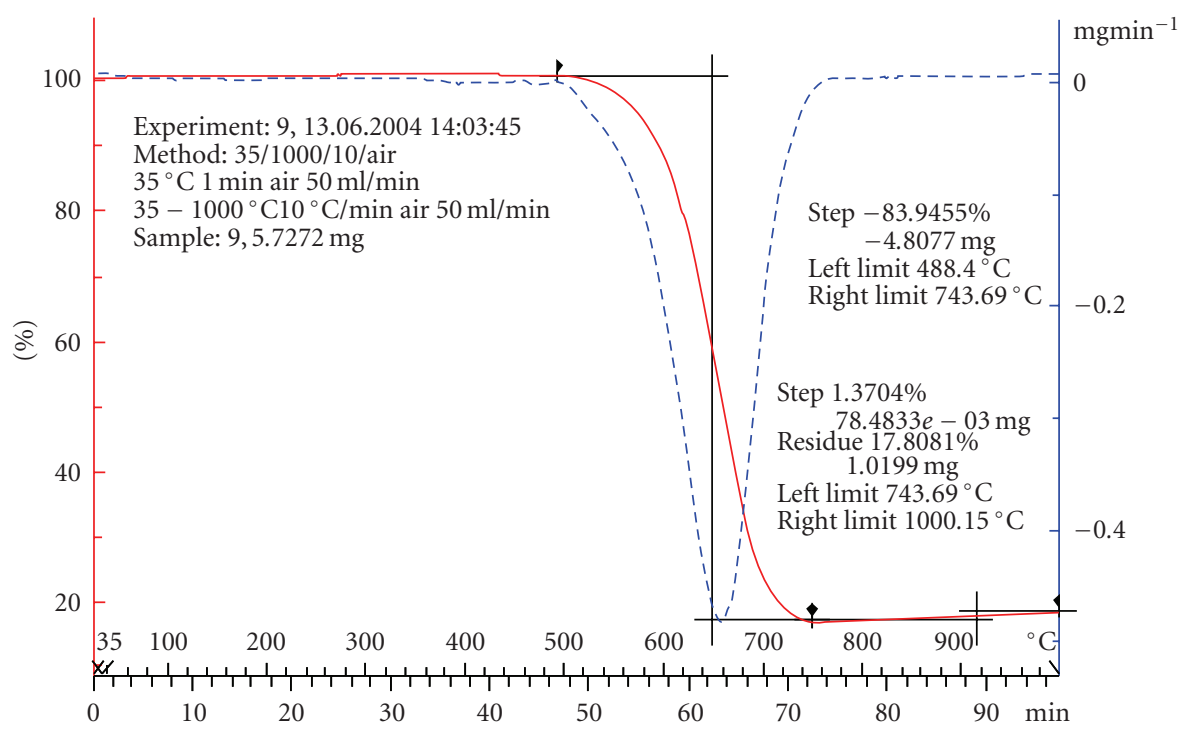

FIgURE 4: Thermogravimetric (TG) curve as red colour Derivative thermogravimetric (DTG) curve as blue colour for pure CNTs.

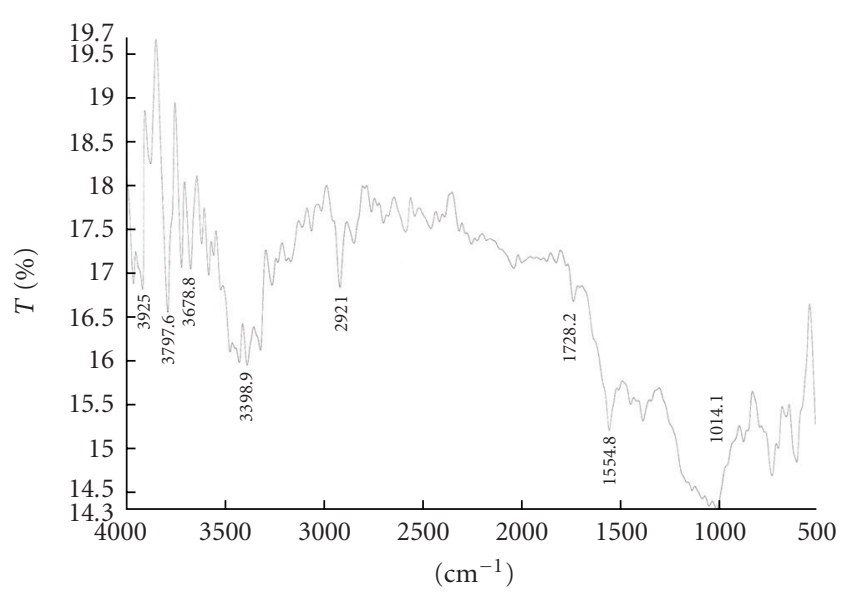

FIGURE 5: FTIR of CNT modified with COOH.

3.4. Effect of $p H$. The removal of chromium by R-CNTs and M-CNTs adsorbents with various $\mathrm{pH}$ has been studied and the results are shown in Figure 6. The variations of $\mathrm{pH}$ used in the experiments are within the range of 4 to 7 . The same phenomenon of the effect of $\mathrm{pH}$ was observed. At acidic $\mathrm{pH}$ medium, free metal ions exist in solution while surface functional groups present in the protonated form. On the other hand, at basic $\mathrm{pH}$ medium, metals are precipitated as their hydroxides while functional groups on adsorbent surface (carboxyl, phenolic, lactonic) exist in the deprotonated form [17]. Therefore, with the increasing of the $\mathrm{pH}$ from 4 to 7 the removal of chromium in the solution will increase and decrease the degree of protonation of the surface which increased the adsorption capacity of the trivalent chromium ion. There was no removal for chromium $\left(\mathrm{Cr}^{3+}\right)$ at $\mathrm{pH} 4$ and $\mathrm{pH} 5$ by using raw CNTs. This happened due to the strong competition of $\mathrm{H}^{+}$with $\mathrm{Cr}^{3+}$ on the adsorption sites. When the $\mathrm{pH}$ increased the

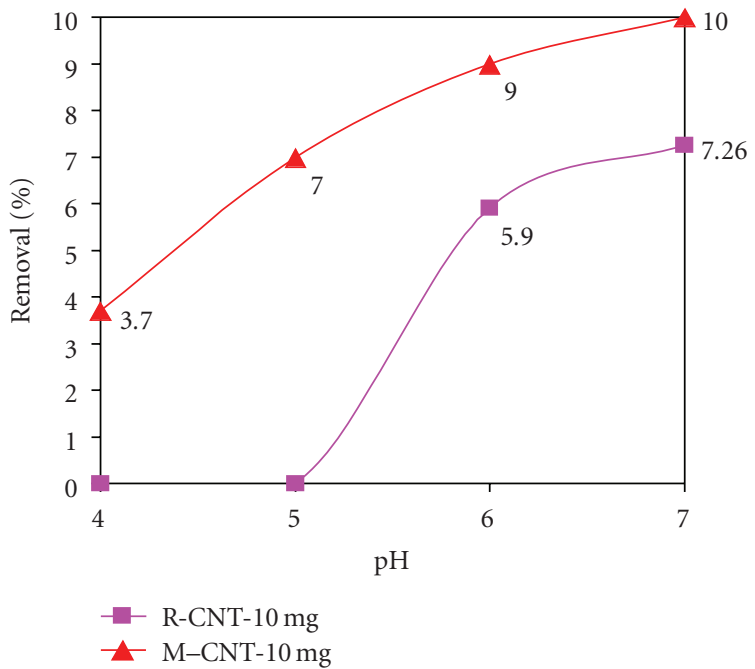

FIGURE 6: The effect of $\mathrm{pH}$ on percentage removal of chromium at $150 \mathrm{rpm}$.

adsorption of chromium increased slightly. At $\mathrm{pH} 6$ the maximum percentage removal of chromium was about 6 percent, and at $\mathrm{pH} 7$ the removal percentage increased to 7.3 percent by using (R-CNTs).The removal increased to only $10 \%$ when (M-CNTs) were used. The weakness of removal of $\mathrm{Cr}^{3+}$ refers to an aqueous solution $\mathrm{Cr}^{3+}$ is in the form $\mathrm{Cr}$ $(\mathrm{OH})^{2+}$ or $\mathrm{CrO}^{-}$. Upon hydration the solid surface develops hydroxyl groups which behave as Bronsted acids according to

$$
\begin{gathered}
\mathrm{CSOH}_{2}{ }^{+} \longleftrightarrow \mathrm{SC}(\mathrm{OH})^{0}+\mathrm{H}^{+}, \\
\mathrm{SC}(\mathrm{OH})^{0} \longleftrightarrow \mathrm{SC}-\mathrm{O}^{-}+\mathrm{H}^{+},
\end{gathered}
$$




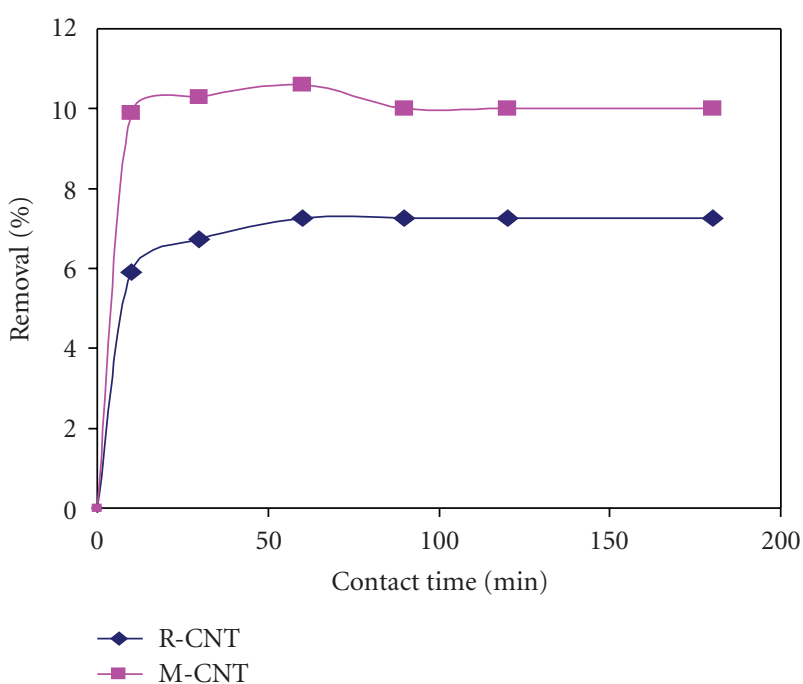

FIgURE 7: The effect of contact time on percentage removal of chromium at $150 \mathrm{rpm}$ at $\mathrm{pH} 7$.

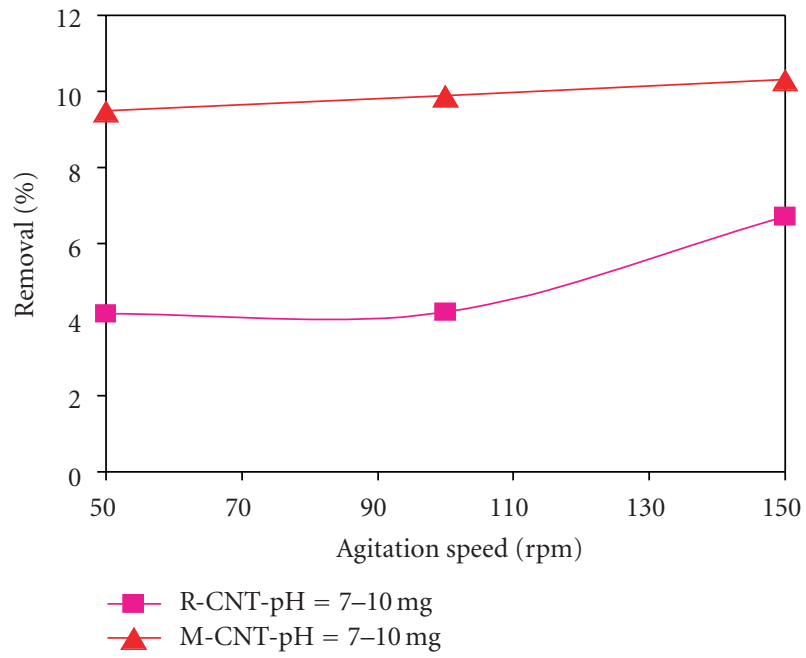

FIGURE 8: The effect of agitation speed on percentage removal of chromium at $\mathrm{pH} 7$.

where CS- $-\mathrm{OH}^{+2}$ represents the protonated surface hydroxyl groups when the solution acidity is below the $\mathrm{pH}_{\mathrm{ZPC}}, \mathrm{SC}-$ $(\mathrm{OH})^{0}$ illustrates the neutral surface at the $\mathrm{pH}_{\mathrm{ZPC}}$ while SC$\mathrm{O}^{-}$is the ionized surface above the $\mathrm{pH}_{\mathrm{ZPC}}$.

3.5. Effect of Contact Time. The adsorption behavior of $\mathrm{Cr}$ by R-CNT and M-CNTs in relation to the effect of contact time was carried out by varying the equilibrium time from 10 minutes to 4 hours at a $\mathrm{Cr}$ concentration of $1 \mathrm{mg} / \mathrm{L}$, a dose of adsorbent of $10 \mathrm{mg} / \mathrm{L}$, and optimum $\mathrm{pH}$ of 7 . The results presented in Figure 7 show that the adsorption rate reach to the equilibrium for the R-CNTs and M-CNTs after one-hour and removal was 7 and 10 percent of $\mathrm{Cr}$, respectively. It is indicating that by suing M-CNTs, the reaction is fast and the adsorption sites are well exposed as compared to R-CNTs, which has low capacity for adsorption. No further increase

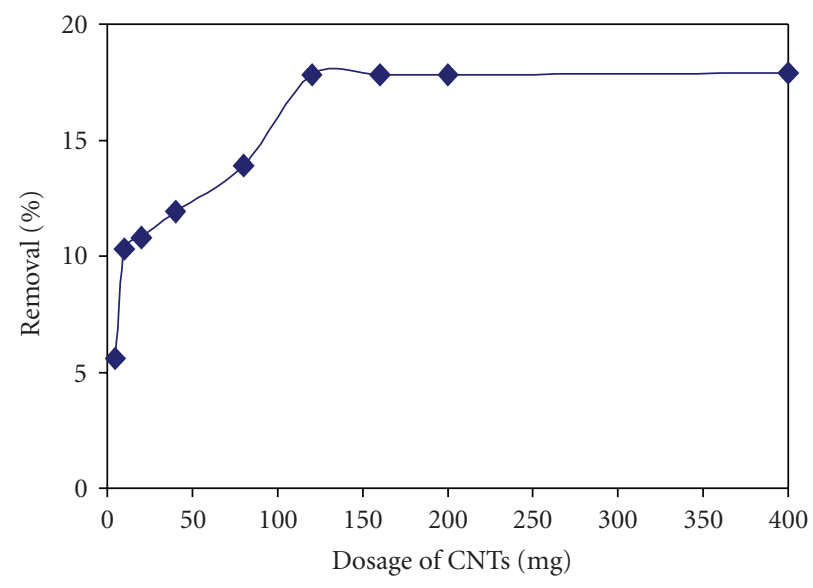

Figure 9: The effect of CNT Dosage on percentage removal of chromium at $\mathrm{pH} 7$.

in removal efficiency was observed with time for R-CNTs and M-CNTs after one-hour.

3.6. Effect of Agitation Speed. The effect of agitation speed on adsorption capacity of chromium has been studied by varying the speed of agitation from 50 to $200 \mathrm{rpm}$ as shown in Figure 8. It has been observed that the percentage of chromium removal increased slightly with increasing agitation speed. Agitation facilitates proper contact between the metal ions in solution and the CNT binding sites and thereby promotes effective transfer of chromium ions to the carbon nanotubes sites. At $50 \mathrm{rpm}$ and $100 \mathrm{rpm}$, the adsorption rates monitored were found to be slightly lower than that at $150 \mathrm{rpm}$. These results indicate that the contact between solids and liquid is more effective at $150 \mathrm{rpm}$. This is due to the fact that, the increase of agitation speed, improves the diffusion of chromium ions towards the surface of the adsorbents.

3.7. Effect of CNTs Dosage. The amount of carbon nanotubes in the water is one of the major factors, which affect the adsorption capacity. The batch adsorption experiments were carried out by using various amounts of M-CNTs from 5 to $400 \mathrm{mg}$ while the $\mathrm{pH}$, agitation speed and contact time were fixed at 7,150 rpm and 120 minutes. The results shown in Figure 9 indicated that the adsorption capacity increased with increased in adsorbent dosage. This is expected because the higher the dose of adsorbents in the solution, the greater the availability of exchangeable sites for ions. This suggests that after a certain dose of adsorbent, the maximum adsorption sets in and hence the amount of ions binds to the adsorbent and the amount of free ions remains constant even with further addition of the dose of adsorbent. Therefore, the adsorption capacity for chromium removal is dependent on the adsorbent dosage. It was found, that the maximum removal of chromium was 20 percent when $120 \mathrm{mg}$ of MCNTs were added. Up to a certain value, no further increase in percent sorption of metal ion occurred as an increase in CNT mass. 


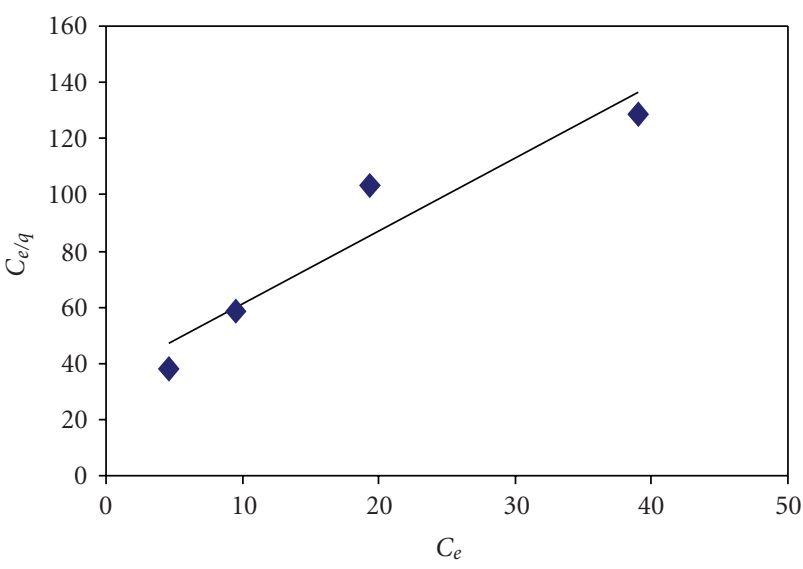

(a)

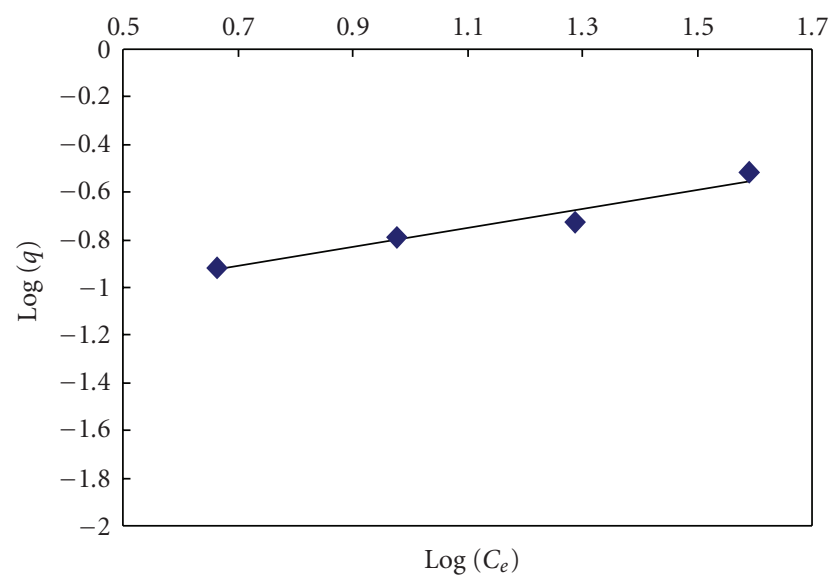

(b)

FIGURe 10: Adsorption isotherm models for chromium: (a) Langmuir and (b) Freundlich.

TABle 1: Parameters of Langmuir and Freundlich adsorption isotherm models for chromium.

\begin{tabular}{lccccc}
\hline & Langmuir & \multicolumn{3}{c}{ Freundlich } \\
$q_{m}$ & $K_{L}$ & $R^{2}$ & $n$ & $K_{F}$ & $R^{2}$ \\
\hline 0.3853 & 0.0741 & 0.9089 & 2.4802 & 0.0642 & 0.9494 \\
\hline
\end{tabular}

3.8. Freundlich and Langmuir Isotherms Models. Freundlich and Langmuir isotherms relate the coverage or adsorption of molecules on a solid surface to gas pressure or concentration of a medium above the solid surface at a fixed temperature. The experimental data for Cr(III) adsorption on CNTs at different $\mathrm{pH}$ values could be approximated by the isotherm models of Langmuir (9) and Freundlich (10)

$$
q=\frac{q_{m} K_{L} C}{1+K_{L} C}
$$

where $C$ is the equilibrium $\mathrm{Cr}(\mathrm{III})$ concentration $(\mathrm{mg} / \mathrm{l}), q$ is the amount adsorbed $(\mathrm{mg} / \mathrm{g})$ and $q_{m}$ and $K_{L}$ are Langmuir constants related to adsorption capacity and energy of adsorption, respectively

$$
q=K_{F} C^{1 / n}
$$

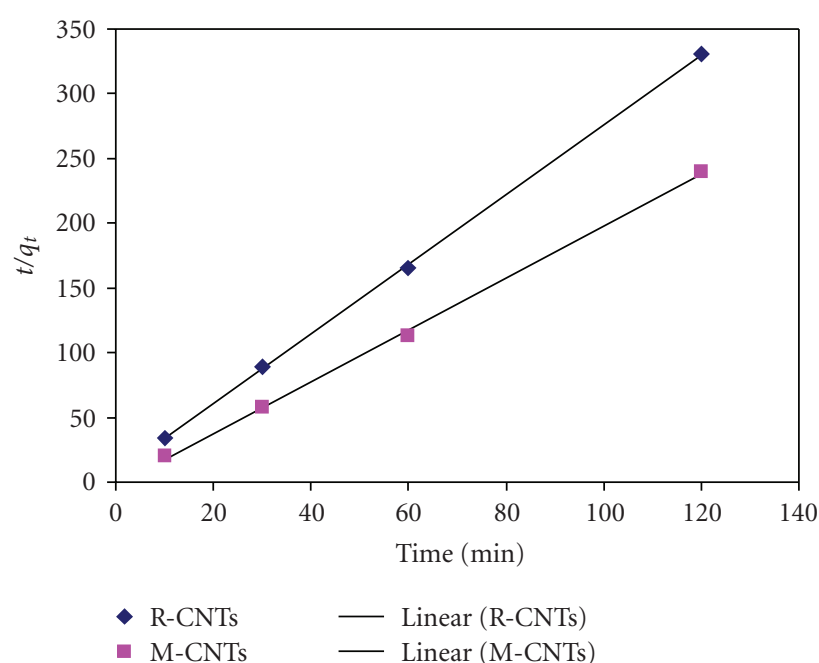

Figure 11: Pseudo-second-order kinetics of Cr (III) using R-CNTs and M-CNTs.

where $K_{F}$ and $n$ are Freundlich constants related to adsorption capacity and adsorption intensity, respectively.

The equations (9) and (10) can be written as

$$
\begin{gathered}
\frac{C}{q}=\frac{1}{\left(K_{L} q_{m}\right)}+\frac{C}{q_{m}}, \\
\log q=\frac{1}{n} \log C+\log K_{F}
\end{gathered}
$$

3.9. Langmuir and Freundlich Adsorption Isotherm Models for Chromium. The equilibrium adsorption is important in the design of adsorption systems. Equilibrium studies in adsorption indicate the capacity of the adsorbent during the treatment process. Taking into account that the percentage removal is the highest at $\mathrm{pH} 7$, thus, the condition was used to further optimize the adsorption process parameters. The equilibrium curve was modeled in Figure 10.

The Langmuir and Freundlich equations were used to describe the data derived from the adsorption of $\mathrm{Cr}$ (III) by CNTs over the entire parameters range studied. Based on Figure 10, the adsorption capacity $\left(q_{e}\right)$ and adsorption intensity was determined from the slope and intercept of the graph, respectively.

By comparison of Langmuir and Freundlich isotherms, Freundlich Isotherm shows better fitting model with higher correlation coefficient $\left(R^{2}=0.9494\right)$ compared to Freundlich Isotherm $\left(R^{2}=0.9087\right)$. Therefore, this indicates the applicability of monolayer coverage of $\mathrm{Cr}$ (III) ions on the surface of the adsorbent. This is due to the fact that, CNTs have greater surface area for metal adsorption. The good correlation coefficient of Freundlich isotherm also indicates that $\mathrm{Cr}$ (III) ions strongly adsorbed to the surface of CNTs. Therefore, it is verified that CNTs have great potential to be a good adsorbent for the removal of $\mathrm{Cr}$ (III) ions in water treatment. 
TABLE 2: Kinetic parameters for pseudo-second-order model of chromium.

\begin{tabular}{lccc}
\hline Adsorbent $(10 \mathrm{mg})$ & $q_{e}(\mathrm{mg} / \mathrm{g})$ & $K_{2}\left(\mathrm{~g} \cdot \mathrm{mg}^{-1} \cdot \mathrm{h}^{-1}\right)$ & $R^{2}$ \\
\hline R-CNTs & 0.3718 & 1.06171 & 0.9997 \\
M-CNTs & 0.5 & 1.9144 & 0.9988 \\
\hline
\end{tabular}

3.10. Modeling of Kinetics Adsorption. Modeling of kinetic data is fundamental for the industrial application of adsorption since it gives information for comparison among different biomaterials under different operational conditions for designing and optimizing operational conditions for pollutant removal from wastewater systems [22].

The kinetics were investigated by using the information obtained from the effect of dosage (dry-weight basis) at $25^{\circ} \mathrm{C}$ at three different time intervals up to 120 minutes. The pseudo first-order kinetic equation was not applicable because $R^{2}$ is small comparing to $R^{2}$ of pseudo-second-order equation. Therefore, the pseudo-second-order equation was used in this study in order to investigate the mechanism of adsorption of $\mathrm{Cr}$ (III) by the CNTs and the potential rate-controlling steps, such as mass transport and chemical reactions

$$
\frac{d q_{t}}{d t}=k_{2}\left(q_{e}-q_{t}\right)^{2}
$$

where $q_{e}$ and $q_{t}$ are the sorption capacity at equilibrium and at time $(\mathrm{mg} / \mathrm{g})$, respectively and $K^{2}$ is the rate constant of the pseudo-second-order sorption $\left(\mathrm{g} \cdot \mathrm{mg}^{-1} \cdot \mathrm{min}^{-1}\right)$. For the boundary conditions $t=0$ to $t=t, q_{t}=0$ and $q_{t}=q_{t}$, the integrated form of (12) becomes:

$$
\frac{1}{q_{e}-q_{t}}=\frac{1}{q_{e}}+k_{2} t .
$$

This has a linear form

$$
\frac{1}{q_{t}}=\frac{1}{k_{2} q_{e}^{2}}+\frac{t}{q_{e}} .
$$

The integrated form of the equation is

$$
\frac{t}{q_{t}}=\frac{1}{h}+\left(\frac{1}{q_{e}}\right) t,
$$

where $h\left(\mathrm{~g} \cdot \mathrm{mg}^{-1} \cdot \mathrm{min}^{-1}\right)$ can be regarded as the initial sorption rate $q_{t} / t \rightarrow 0$, hence $h=k_{2} q_{e}^{2}$.

If the pseudo-second-order kinetics is applicable to the experimental data, the plot of $t / q_{e}$ versus time of (15) gives a linear relationship from which $q_{e}, k$, and $h$ can be determined from the slope and intercept of the plot, respectively.

3.11. Kinetics Adsorption Model of Chromium (III). The kinetics adsorption model has been done for chromiume (III) at $\mathrm{pH} 7$ to avoid forming chromium complexation that leads to precipitation. The parameters of modeling are shown in Table 2.

By plotting of $t / q^{t}$ versus time (Figure 11) yields very good straight lines (correlation coefficient, $R^{2}=0.9997$ for R-CNTs and $R^{2}=0.9988$ for M-CNTs. The second order-rate constant obtained from this figure are 1.06171 for R-CNTs and $1.9144\left(\mathrm{~g} \cdot \mathrm{mg}^{-1} \cdot \mathrm{h}^{-1}\right)$ for M-CNTs. The second order rate constant indicates that time to achieve equilibrium concentration of $\mathrm{Cr}$ (III) is less by using MCNTs compare with R-CNTs. The equilibrium adsorption capacity, $q_{e}$ obtained from the graph also implies that $\mathrm{M}$ CNTs have higher adsorption capacity $\left(q_{e}=0.5 \mathrm{mg} / \mathrm{g}\right)$ is compared to R-CNTs $\left(q_{e}=0.3718 \mathrm{mg} / \mathrm{g}\right)$.

\section{Conclusion}

Carbon Nanotubes were found to be efficient for the adsorption of $\mathrm{Cr}$ (III) in aqueous solution. The characterization of $\mathrm{Cr}$ (III) uptake showed that, the Cr (III) binding is dependent on initial $\mathrm{pH}$, agitation speed, amount of dosage, and contact time. Percentage uptake increased with an increased in $\mathrm{pH}$ from $\mathrm{pH} 4$ to $\mathrm{pH}$ 7. The optimum $\mathrm{pH}$ found in this study is $\mathrm{pH} 7$ in which it gave 6\% removal of $\mathrm{Cr}$ (III) ions by using R-CNTs and $10 \%$ of Cr (III) ions by using M-CNTs from aqueous solution. The percentage uptake increase slightly with an increase in agitation speed from 50 to $150 \mathrm{rpm}$, in which $150 \mathrm{rpm}$ gave slightly higher removal for chromium. While the percent removal of Cr (III) was observed to be optimal for higher dosage of CNTs, in which $150 \mathrm{mg}$ of MCNTs contribute to $18 \%$ removal of $\mathrm{Cr}$ (III).

\section{Acknowledgment}

The authors would like to acknowledge the support provided by King Abdulaziz City for Science and Technology (KACST) through Science \& Technology Unit at King Fahd University of petroleum \& Minerals (KFUPM) for funding this work through project no. 08-NAN91-4 as part of the National Science, Technology, and Innovation Plan.

\section{References}

[1] G. F. Nordberg, B. Flower, M. Nordberg, and L. Friberg, Handbook on the Toxicology of Metals, Academic Press, New York, NY, USA, 3rd edition, 2007.

[2] M. M. Rao, A. Ramesh, G. P. C. Rao, and K. Seshaiah, "Removal of copper and cadmium from the aqueous solutions by activated carbon derived from Ceiba pentandra hulls," Journal of Hazardous Materials, vol. 129, no. 1-3, pp. 123-129, 2006.

[3] M. Sekar, V. Sakthi, and S. Rengaraj, "Kinetics and equilibrium adsorption study of lead(II) onto activated carbon prepared from coconut shell," Journal of Colloid and Interface Science, vol. 279, no. 2, pp. 307-313, 2004.

[4] J. Ayala, F. Blanco, P. Garcia, P. Rodriguez, and J. Sancho, "Asturian fly ash as a heavy metals removal material," Fuel, vol. 77, no. 11, pp. 1147-1154, 1998.

[5] Y. S. Ho and G. McKay, "The sorption of lead(II) ions on peat," Water Research, vol. 33, no. 2, pp. 578-584, 1999.

[6] W. Chu, "Lead metal removal by recycled alum sludge," Water Research, vol. 33, no. 13, pp. 3019-3025, 1999.

[7] P. Brown, I. A. Jefcoat, D. Parrish, S. Gill, and E. Graham, "Evaluation of the adsorptive capacity of peanut hull pellets 
for heavy metals in solution," Advances in Environmental Research, vol. 4, no. 1, pp. 19-29, 2000.

[8] C. V. Diniz, F. M. Doyle, and V. S. T. Ciminelli, "Effect of pH on the adsorption of selected heavy metal ions from concentrated chloride solutions by the chelating resin Dowex M-4195," Separation Science and Technology, vol. 37, no. 14, pp. 31693185, 2002.

[9] M. Arias, M. T. Barral, and J. C. Mejuto, "Enhancement of copper and cadmium adsorption on kaolin by the presence of humic acids," Chemosphere, vol. 48, no. 10, pp. 1081-1088, 2002.

[10] R. Sublet, M.-O. Simonnot, A. Boireau, and M. Sardin, "Selection of an adsorbent for lead removal from drinking water by a point-of-use treatment device," Water Research, vol. 37, no. 20, pp. 4904-4912, 2003.

[11] B. Biskup and B. Subotic, "Removal of heavy metal ions from solutions using zeolites. III. Influence of sodium ion concentration in the liquid phase on the kinetics of exchange processes between cadmium ions from solution and sodium ions from Zeolite A," Separation Science and Technology, vol. 39, no. 4, pp. 925-940, 2004.

[12] Q. Li, S. Wu, G. Liu, et al., "Simultaneous biosorption of cadmium (II) and lead (II) ions by pretreated biomass of Phanerochaete chrysosporium," Separation and Purification Technology, vol. 34, no. 1-3, pp. 135-142, 2004.

[13] F. Ekmekyapar, A. Aslan, Y. K. Bayhan, and A. Cakici, "Biosorption of copper(II) by nonliving lichen biomass of Cladonia rangiformis hoffm," Journal of Hazardous Materials, vol. 137, no. 1, pp. 293-298, 2006.

[14] G. P. Rao, C. Lu, and F. Su, "Sorption of divalent metal ions from aqueous solution by carbon nanotubes: a review," Separation and Purification Technology, vol. 58, no. 1, pp. 224231, 2007.

[15] S. Iijima, "Helical microtubules of graphitic carbon," Nature, vol. 354 , no. 6348 , pp. 56-58, 1991.

[16] S. Haddon, "Carbon nanotubes," Accounts of Chemical Research, vol. 35, no. 12, pp. 977-1113, 2002.

[17] A. H. El-Sheikh, "Effect of oxidation of activated carbon on its enrichment efficiency of metal ions: comparison with oxidized and non-oxidized multi-walled carbon nanotubes," Talanta, vol. 75, no. 1, pp. 127-134, 2008.

[18] Y.-H. Li, J. Ding, Z. Luan, et al., "Competitive adsorption of $\mathrm{Pb}^{2+}, \mathrm{Cu}^{2+}$ and $\mathrm{Cd}^{2+}$ ions from aqueous solutions by multiwalled carbon nanotubes," Carbon, vol. 41, no. 14, pp. 2787-2792, 2003.

[19] S.-H. Hsieh and J.-J. Horng, "Adsorption behavior of heavy metal ions by carbon nanotubes grown on microsized $\mathrm{Al}_{2} \mathrm{O}_{3}$ particles," Journal of University of Science and Technology Beijing, Mineral, Metallurgy, Material, vol. 14, no. 1, pp. 7784, 2007.

[20] M. I. Kandah and J.-L. Meunier, "Removal of nickel ions from water by multi-walled carbon nanotubes," Journal of Hazardous Materials, vol. 146, no. 1-2, pp. 283-288, 2007.

[21] D. Xu, X. Tan, C. Chen, and X. Wang, "Removal of Pb(II) from aqueous solution by oxidized multiwalled carbon nanotubes," Journal of Hazardous Materials, vol. 154, no. 1-3, pp. 407-416, 2008.

[22] A. Nassereldeen, A. A. Muataz, A. Abdullah, E. S. Mohamed, M. D. Alam, and N. Yahya, "Kinetic adsorption of application of carbon nanotubes for $\mathrm{Pb}(\mathrm{II})$ removal from aqueous solution," Journal of Environmental Sciences, vol. 21, pp. 539-544, 2009.

[23] A. A. Muataz, G. Nazlia, M. El-Sadig, et al., "Effect of multi wall carbon nanotubes (MWCNTS) on the mechanical properties of natural rubber," Journal of Fullerenes, Nanotubes and Carbon Nanostructures, vol. 14, no. 4, pp. 641-649, 2006.

[24] A. A. Muataz, F. Ahmadun, C. Guan, E. Mahdi, and A. Rinaldi, "Effect of reaction temperature on the production of carbon nanotube," NANO: Brief Reports and Reviews, vol. 1, no. 3, pp. 251-257, 2006.

[25] G. Nazlia, A. Fakhru'l-Razi, A. R. Suraya, and A. A. Muataz, "Multi-wall carbon nanotubes/styrene butadiene rubber (SBR) nanocomposite," Journal of Fullerenes, Nanotubes and Carbon Nanostructures, vol. 15, no. 3, pp. 207-214, 2007.

[26] A. A. Muataz, N. Nazif, Y. Faridah, et al., "Radiation vulcanization of natural rubber latex loaded with carbon nanotubes," Fullerenes, Nanotubes and Carbon Nanostructures, vol. 18, no. 1, pp. 56-71, 2010.

[27] A. A. Muataz, M. Fettouhi, A. Al-Mammum, and N. Yahya, "Lead removal by using carbon nanotubes," International Journal of Nanoparticles, vol. 2, no. 1-6, pp. 329-338, 2009.

[28] S. Goyanes, G. R. Rubiolo, A. Salazar, A. Jimeno, M. A. Corcuera, and I. Mondragon, "Carboxylation treatment of multiwalled carbon nanotubes monitored by infrared and ultraviolet spectroscopies and scanning probe microscopy," Diamond \& Related Materials, vol. 16, no. 2, pp. 412-417, 2007.

[29] J. Zhang, H. Zou, Q. Qing, et al., "Effect of chemical oxidation on the structure of single-walled carbon nanotubes," Journal of Physical Chemistry B, vol. 107, no. 16, pp. 3712-3718, 2003. 

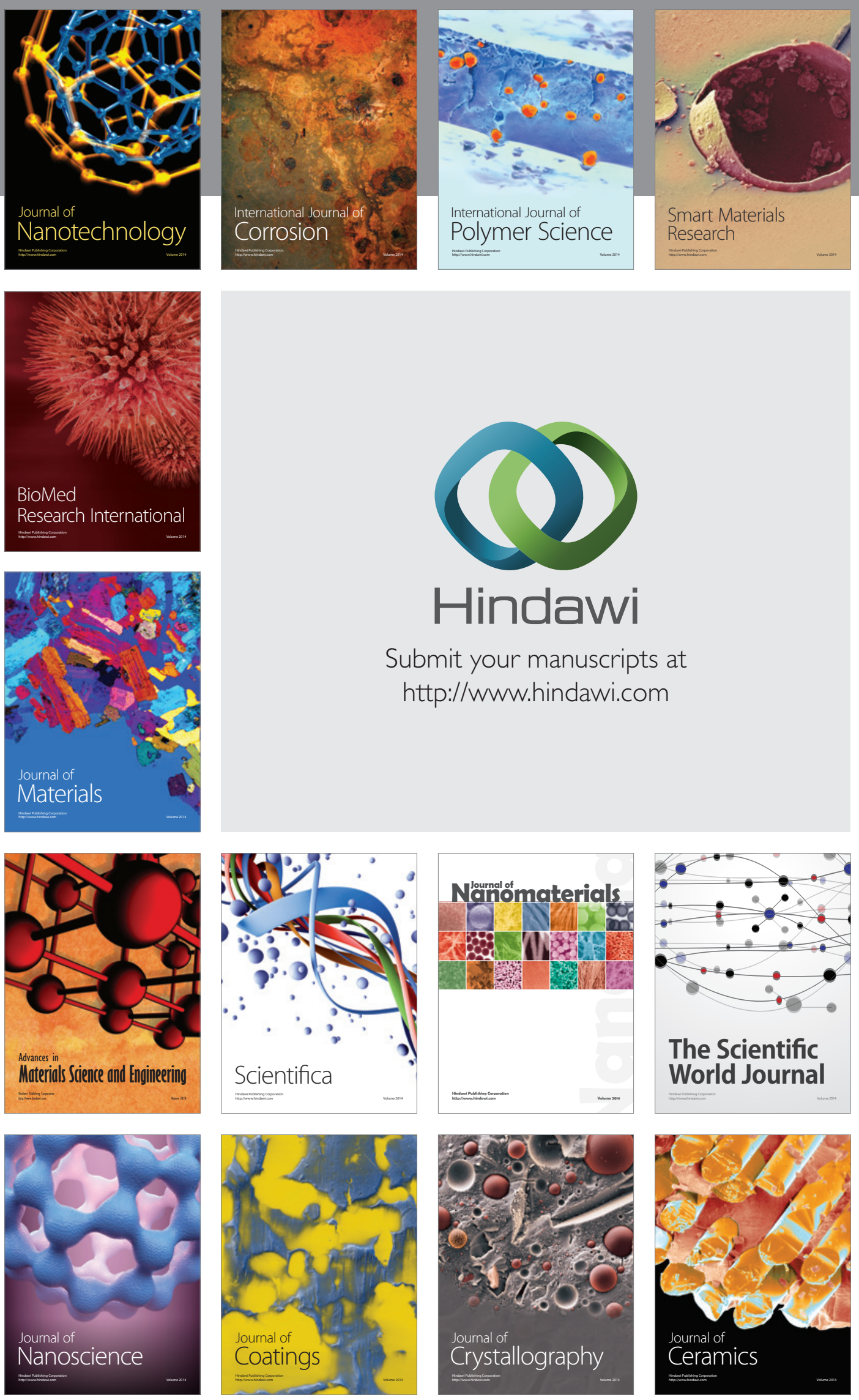

The Scientific World Journal

Submit your manuscripts at

http://www.hindawi.com

\section{World Journal}

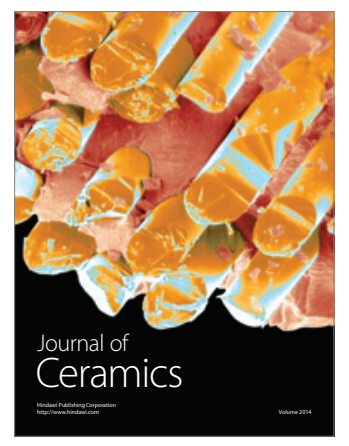

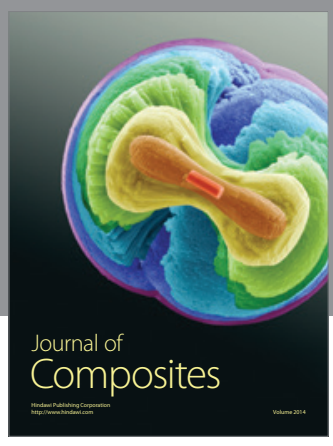
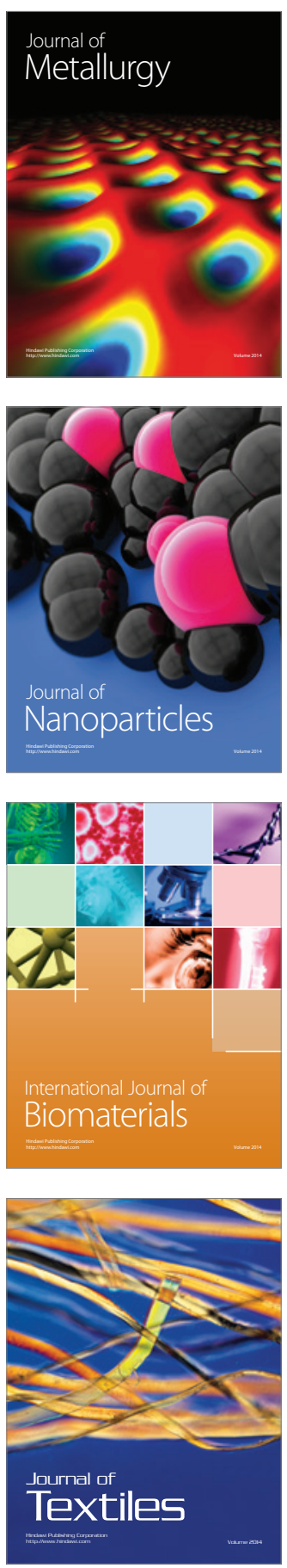